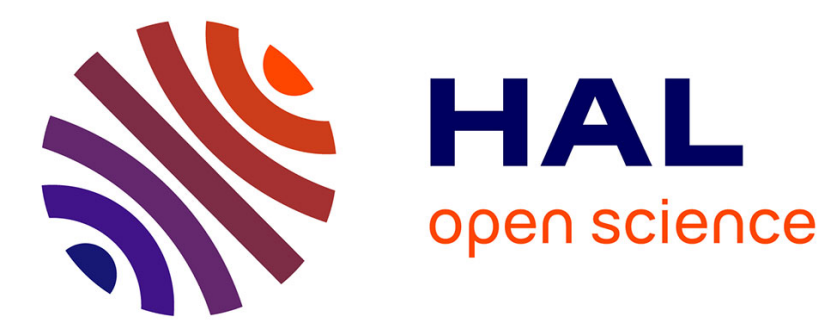

\title{
Evidence for Local Orientational Order in Salt-Free Worm-Like Micelles: A Transient Electric Birefringence Study
}

\author{
R. Oda, F. Lequeux, E. Mendes
}

\section{- To cite this version:}

R. Oda, F. Lequeux, E. Mendes. Evidence for Local Orientational Order in Salt-Free Worm-Like Micelles: A Transient Electric Birefringence Study. Journal de Physique II, 1996, 6 (10), pp.14291439. 10.1051/jp2:1996140 . jpa-00248379

\section{HAL Id: jpa-00248379 https://hal.science/jpa-00248379}

Submitted on 1 Jan 1996

HAL is a multi-disciplinary open access archive for the deposit and dissemination of scientific research documents, whether they are published or not. The documents may come from teaching and research institutions in France or abroad, or from public or private research centers.
L'archive ouverte pluridisciplinaire HAL, est destinée au dépôt et à la diffusion de documents scientifiques de niveau recherche, publiés ou non, émanant des établissements d'enseignement et de recherche français ou étrangers, des laboratoires publics ou privés. 


\title{
Evidence for Local Orientational Order in Salt-Free Worm-Like Micelles: A Transient Electric Birefringence Study
}

\author{
R. Oda $\left(^{*}\right)$, F. Lequeux and E. Mendes \\ Laboratoire d'Ultrasons et de Dynamique des Fluides Complexes $\left({ }^{* *}\right)$, (L.U.D.F.C), \\ 4 rue Blaise Pascal, 67070 Strasbourg Cedex, France
}

(Received 1 April 1996, received in final form 14 June 1996, accepted 2 July 1996)

PACS.61.30.Gd - Orientational order of liquid crystals; electric and magnetic fields effects on
order
PACS.61.25.Hq - Macromolecular and polymer solutions; polymer melts; swelling

\begin{abstract}
Transient electric birefringence experiments were performed on a aqueous dispersions of salt-free charged giant micelles of a recently introduced gemini surfactant (referred as 12-2-12). In the dilute regime, the system consists of small rod-like micelles, while at higher concentrations (semi-dilute region) the micelles are semi-flexible and entangled. For concentrations far from $c^{*}$, either dilute or semi-dilute regime, the birefringence has the same sign suggesting that the micelles align with the electric field in the same direction in both dilute and entangled regime. For concentrations close to $c^{*}$, an anomalous signal with an opposite sign is observed. For the entire surfactant concentration range studied here, we show that the static signals of the electric birefringence can be split into three different components with very different characteristic times, each component being associated to specific orientational mechanism: 1) a parallel alignment to the applied electric field after free rotation in the dilute regime, or confined rotation of small segments in the semi-dilute regime, 2) an alignment perpendicular to the field that appears near $c^{*}$, and interpreted as the result of pre-smectic ordering as recently proposed by Cates, 3) a parallel alignment after disentanglement occurring in the semi-dilute regime.
\end{abstract}

\section{Introduction}

Surfactant molecules dissolved in various solvents can self-assemble reversibly into spatially organised structures forming associations which are physically and not chemically bound. Under certain conditions, they form long flexible worm-like micelles of size depending strongly on temperature, surfactant and salt concentrations [1]. Most of the study concerning structural and dynamic behaviours of these worm-like micellar solutions were focused on systems with presence of a large amount of salt [2]. However, during the last few years, new surfactant systems have been introduced $[3,4]$, and it is now possible to obtain the worm-like micellar phase in the absence of added salt for some ionic surfactants [5]. This allows us to investigate the electrostatic interactions which strongly influence the structures and the dynamics of the surfactant self-assemblies. The surfactant used here is the ethylene-bis (dodecyldimethylam-

(*) Author for correspondence (e-mail: reiko@fresnel.u-strasbg.fr)

$\left({ }^{* *}\right)$ Laboratoire associé au C.N.R.S., URA 851 
monium bromide) shortly called as "12-2-12" introduced by Zana et al. [4], where the direct evidence for the existence of long cylindrical micelles had been obtained by cryo-TEM. It has been shown $[6,7]$ that, in the dilute regime, (for the studied system, less than $c^{*} \equiv 0.9 \%$ (wt)) these charged surfactants assemble in rigid rods, their size being limited by electrostatic repulsion. Beyond the overlapping concentration, $c^{*}$, the micelles become entangled, and grow exponentially with concentration due to the screening of intermicellar electrostatic interaction. In this semi-dilute regime, they behave similarly to polyelectrolyte polymers [2].

Time resolved electric birefringence allows one to probe the angular dynamics of suspended assemblies in solution. Those techniques have recently been applied to the study of polyelectrolytes [8] and worm-like micellar solutions [9] by Hoffmann's group, where they brought to evidence an anomalous behaviour for concentrations near $c^{*}$. A theoretical interpretation of such an anomaly was developed by Cates [10]. He proposed that, when an external electric field is applied to a polyelectrolyte solution near $c^{*}$, the chains tend to align perpendicular to the field due to local pre-smectic ordering.

In the work presented here, we report results on the rotational dynamics of 12-2-12 micellar solutions using transient electric Birefringence (TEB). In the absence of salt, we have investigated solutions in dilute and semi-dilute regimes, as well as around the overlap concentration, $c^{*}$, where an anomalous behaviour was found as in another worm-like micellar system $[8,9]$. We also report below the dependence of the TEB signal as a function of the temperature for some surfactant concentrations. Moreover, we were able to split the signal into three contributions which originate in three different orientational mechanisms. This allows us to give a complete interpretation of the quite complex dynamic behaviour of the micelles probed by the TEB experiments.

\section{Experimental Section}

The transient electric birefringence measurements were performed with a He-Ne laser $(\lambda=$ $6238 \AA$ ), polarised at $45^{\circ}$ with respect to vertical. The laser beam passed through a thermostated cell containing the solution between two steel electrodes which were placed parallel to each other with a separation of $2 \mathrm{~mm}$. The electric field is applied perpendicular to the laser beam and parallel to the optical table. After the sample cell, a $\lambda / 4$ plate and an analyser (crossed with the polariser) are placed before a photodiode. A part of the laser beam is taken before the polariser and used to analogically subtract the laser noise from the measurement. During the experiment, a square tension pulse, up to $900 \mathrm{~V}$, can be applied on the electrodes. The applied tension changes sharply from 0 to $900 \mathrm{~V}$ (or from $900 \mathrm{~V}$ to 0 ) in a timescale of the order of $0.5 \mu \mathrm{s}$. The overall time resolution of the set up is about $1 \mu \mathrm{s}$.

The surfactant molecules were synthesised by Zana (Insitut Charles Sadron, CNRS, Strasbourg, France) [3], and the samples were studied in heavy water. Repetitive application of the electric pulses causes electroconvection, which leads to inhomogeneities as well as changes of index of refraction of the sample, which cause the shift of the baseline of the spectra before and after the pulse. Therefore each pulse was applied after at least 15 minutes interval, and samples were replaced frequently. Various concentrations were studied around the transition between dilute and semi-dilute regimes, starting from $0.4 \%$ and up to $1.8 \%$ (weight $\%$ ). Samples were kept in the oven $\left(40^{\circ} \mathrm{C}\right)$ for at least one day after the preparation before measurements to achieve complete thermal equilibrium, and measurements were performed within one month. 

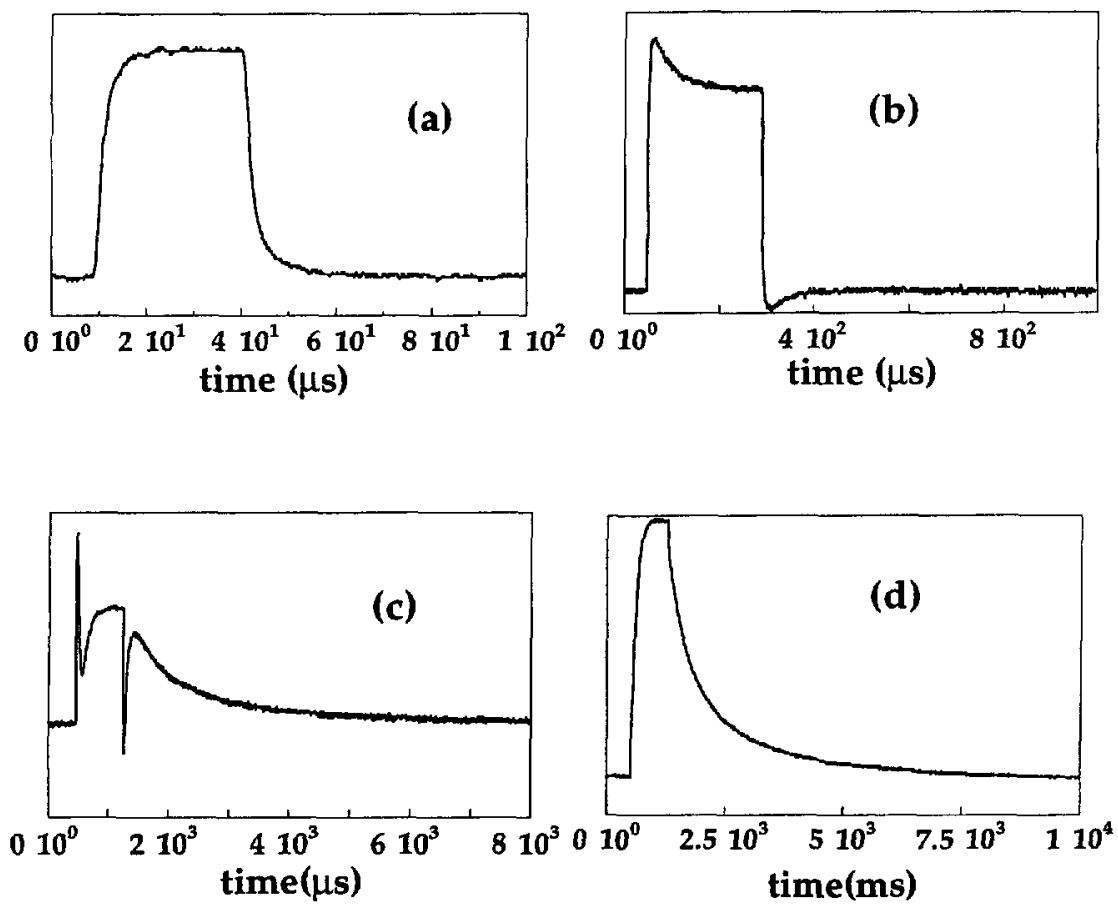

Fig. 1. - Four types of birefringence signals for high applied fields at $21^{\circ} \mathrm{C}$; for different surfactant concentrations: (a) $0.6 \%$, (b) $0.8 \%$, (c) $1.0 \%$, (d) $1.8 \%$ (\% weight).

\section{Results and Discussion}

We describe below the dependence of the static birefringence of 12-2-12 solutions in the concentration ranging from the dilute to the semi-dilute regimes, as well as its dependence on temperature.

3.1. Dependence of the Birefringence on Surfactant Concentration. - The TeB signals for different concentrations for $T=21{ }^{\circ} \mathrm{C}$ are shown in Figure 1. These static birefringence signals show a large variation, and as can be seen, they can be separated into three different components with different characteristic times.

(1) In the dilute regime, $c=0.6 \%$, the signal is positive where the characteristic time is of the order of $5 \mu \mathrm{s}$.

(2) As the concentration increases, a negative component appears at the concentration about $0.8 \%$. This negative signal is superimposed on the positive signal, and it has the typical characteristic time on the order of $50 \mu \mathrm{s}$, ten times slower than the previous one.

(3) As the concentration is further increased, a third and positive signal, with a much longer characteristic time $(500 \mu \mathrm{s}$ or longer) appears at concentrations which are around $1 \%$. This very slow component is also superimposed on the previous ones. Let us remark that if the pulse is cut where the plateau of the second component is achieved, one might miss the rise of the third positive signal.

(4) With the further increase of the surfactant concentration, $c \geq 1.5 \%$, the negative signal disappears, but the very fast first component and very slow one (both positive) remain observable. 


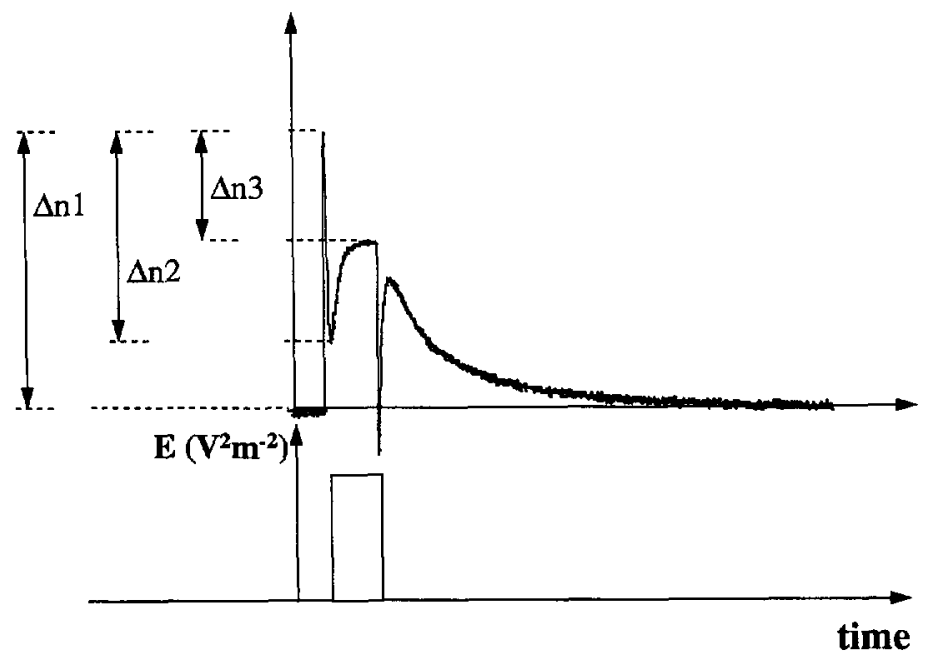

Fig. 2. - Splitting of the birefringence signal into three components $\Delta n_{\imath}(i=1,2,3)$. This splitting procedure applies for any of the four different spectra shown in Figure 1.

Since the characteristic times of the three processes described above differ from each other by roughly an order of magnitude, the static signals can be directly split into three components. A typical decomposition is shown in Figure 2, where the static components of the $\Delta n_{\imath}(i=1,2,3)$ are defined. The indexes $i=1,2,3$ correspond to the first, second and third components, respectively. Those three components are plotted separately in Figure 3 as a function of the square of the applied electric field, $E^{2}$, for different surfactant concentrations. It has to be emphasised that this is different from extracting the plateau height with respect to the base line as classically done $[8,9]$. As can be seen in Figure 3, the dependence of the positive components of the static birefringence, $\Delta n_{1}$, as a function of the electric field, $E$, follows Kerr's law, varying linearly with $E^{2}$. However, both $\Delta n_{2}$ and $\Delta n_{3}$ exhibit a non-linear behaviour with $E^{2} . \Delta n_{2}$ first increases with $E^{2}$ but saturates and decreases for higher values of the field. $\Delta n_{3}$ saturates for quite low values of $E$.

Consider separately the dependence of each component, $\Delta n_{2}(i=1,2,3)$, of the static birefringence on surfactant concentration in the limit of small fields around the overlap concentration. Parallel rheological and light scattering measurements [4] have shown that the overlap concentration, $c^{*}$, was around $0.8 \sim 1.0 \%$ (wt.) surfactant weight. Here, the concentration ranges from $0.4 \%$ (dilute) to $1.8 \%$ (semi-dilute). In Figure $4 \Delta n_{\imath}(\imath=1,2,3)$ are displayed as a function of concentration for a fixed value of $E=850 \mathrm{~V} / \mathrm{m}$. Herein, the origin of each contribution is discussed separately.

\section{- $\Delta n_{1}$ : The Rapid Positive Response to the Applied Field}

This rapid $(\sim 5 \mu \mathrm{s})$ positive signal was observed for the entire range of concentration studied here. For dilute samples at low fields, the signal increases linearly to the surfactant concentration, while for concentrations higher than $1 \%$, this signal starts decreasing with increasing concentration. The micellar length at the critical concentration can be estimated by packing arguments to be about $50 \mathrm{~nm}$ if one assumes that the surfactant molecular length is $\sim 2 \mathrm{~nm}$ 

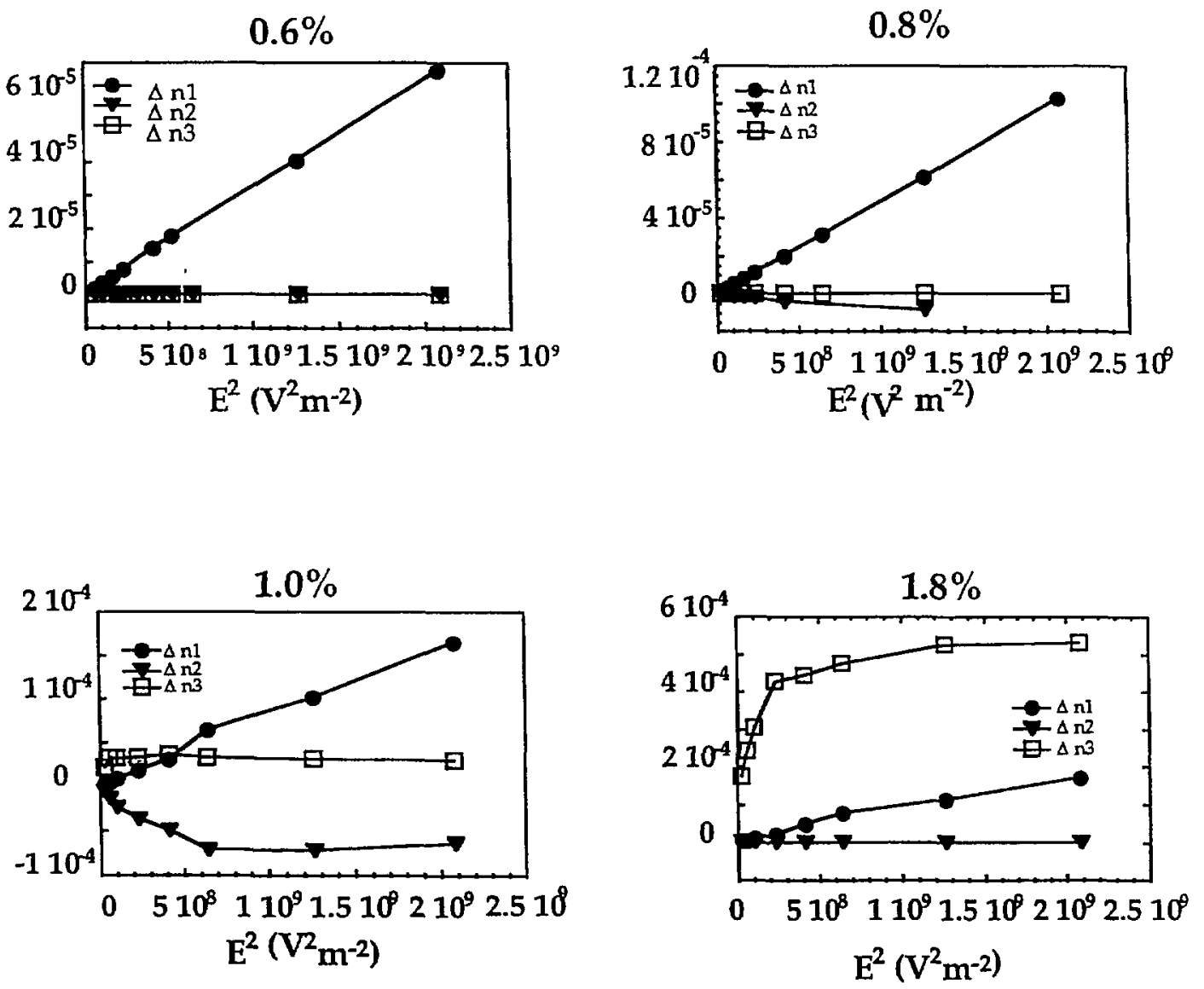

Fig. 3. - Variation of $\Delta n_{\imath}(i=1,2,3)$ as a function of the square of the applied field, $E^{2}$.

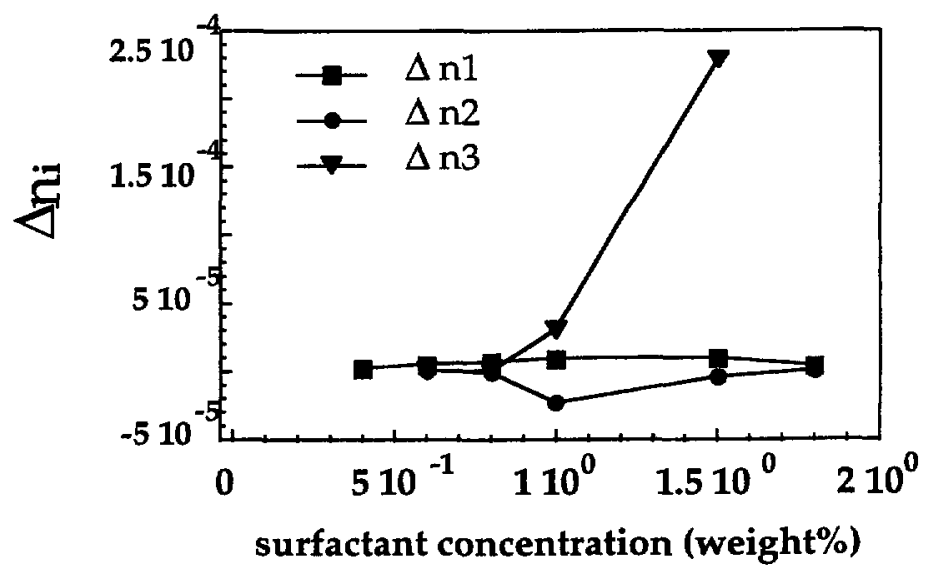

Fig. 4. - Variation of $\Delta n_{\imath}(i=1,2,3)$ as function of surfactant concentration, for a small value of the electric field. 
and that the average surface area per polar head is $0.65 \mathrm{~nm}^{2}$. Using crude thermodynamic estimation, it can be shown that micelles of length $\sim 30 \mathrm{~nm}$ have a rotational time of $5 \mu \mathrm{s}$. Therefore, for $c<c^{*}$, the signal $\Delta n_{1}$ can be associated to free rotation of isolated micelles. The increase of the signal $\Delta n_{1}$ with concentration corresponds to the fact that the signal is proportional to the density of micelles, as well as their length. This variation is small since the length of a micelle in the dilute regime varies only logarithmically with the surfactant concentration [6].

In the semi-dilute regime, for $c \geq c^{*}$, micelles are entangled, and most of them cannot rotate freely because of steric interactions (or entanglements). Two different mechanisms may be responsible for the $\Delta n_{1}$ signal in this regime. First, these long entangled micelles can still partly rotate before being sterically blocked by their neighbours. Secondly, because of the large polydispersity due to the breaking-recombining mechanism, there are always some micelles which are smaller than the mesh size. They can in principle rotate freely. However, as the concentration increases in the semi-dilute regime, the average length of micelles grows and the mesh size diminishes, leading to a decrease of the population of free micelles [7]. At the same time, because of the smaller mesh size, the local rotation of entangled micelles becomes even more restricted.

The combination of the effects described above leads to a maximum for $\Delta n_{1}$ around $c^{*}$. This effect is more clearly displayed in Figure 5a, where each component of the static birefringence is plotted separately.

\section{- $\Delta n_{2}$ : The Slow Negative Response to the Applied Field}

In Figures 4 and $5 \mathrm{~b}, \Delta n_{2}$ as a function of surfactant concentration is plotted at $T=20{ }^{\circ} \mathrm{C}$. This negative contribution to the static birefringence exists only in a certain concentration range, around $c^{*} \sim 1.0 \%$, and is observed neither for dilute nor semi-dilute regimes far from $c^{*}$. In Figure 6. the dependence of $\Delta n_{2}$ on $E^{2}$ is plotted for different temperatures for a fixed surfactant concentration $1.5 \%$. Around this concentration, the amplitude of this negative birefringence increases linearly with $E^{2}$ for low fields. For certain temperatures, we can observe that it saturates (and decreases) at higher field values.

This anomalous signal was first observed by Hoffmann $[8,9]$ in polyelectrolyte solutions and worm-like micellar solution. Recently, Cates [10] proposed an interpretation for the case of polyelectrolyte solutions in which the chains possess many statistical units and form rod-like assemblies. Although, unlike polyelectrolyte, micelles possess only few statistical units near $c^{*}$, due to their long persistence length, they themselves are rod-like around this concentration. The overall qualitative behaviour described by Cates can thus be applied to micellar systems. His argument states the following: at concentrations close to $c^{*}$, there would exist a strong local coupling between the orientation of neighbouring pairs of rod-like micelles. Non-entangled micelles are, therefore, likely to be parallel to one another and form disc-like clusters. Since, for the case of micelles, the nematic long-range ordering would be energetically unfavorable, a local disc-like ordering would be favoured. When the electric field is applied to such a "presmectic clustering" [10], in order not to break this local ordering, (which would require the energy) the macroscopic polarisability would be higher (while the energy would be lower) for the configuration where the induced dipole of each micelle is aligned perpendicular to the long axis of a micelle (in the plane of the "disc"). This would lead to a birefringence signal opposite in sign to the one found in the dilute regime. Thus, that we observed a negative contribution just around $c^{*}$ is consistent with this argument: if the solution is too dilute, there is no more orientational interaction, and if the solution is concentrated above $c^{*}$, micellar length increases 
a)

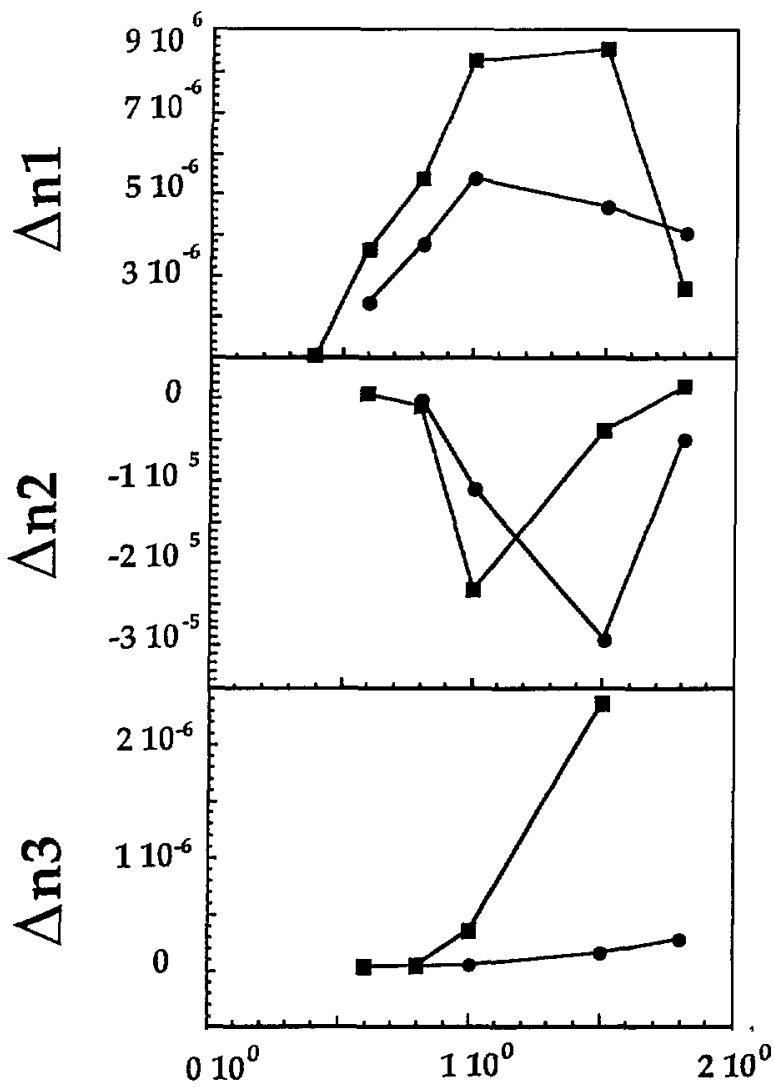

surfactant concentration (weight $\%$ )

Fig. 5. - Variation of a) $\Delta n_{1}$ b) $\Delta n_{2}$ c) $\Delta n_{3}$ as functions of the surfactant concentration. Two different temperatures are considered: $20^{\circ} \mathrm{C}(\boldsymbol{\square})$ and $30{ }^{\circ} \mathrm{C}(\bullet)$.

rapidly with the concentration $[6,7]$, leading to the entanglement of micelles which become much longer than their persistent length. Therefore, the disk-like ordering does not exist for the concentrations far from $c^{*}$.

Using the Stokes-Einstein equation and the measured characteristic time for the anomalous component $\left(\sim 50 \mu \mathrm{s}\right.$ at $20^{\circ} \mathrm{C}$ ) one can estimate the dimension associated to such a domain (the radius of a disk) to be about $1100 \AA$. If we assume the aspect ratio of these micelles to be about $2[10]$, at $c^{*}(L \sim 500 \AA)$, the number of micelles in such domain will be of the order of 15 .

Conversely, as can be seen from Figure $6, \Delta n_{2}$ tends to vanish as high electric fields are applied $\left(T=20^{\circ} \mathrm{C}\right.$ and $28{ }^{\circ} \mathrm{C}$ ). This suggests that any domain or structure responsible for the anomalous signal is destroyed when the external field is strong enough. This observation is consistent with the pre-smectic ordering picture [10]. 


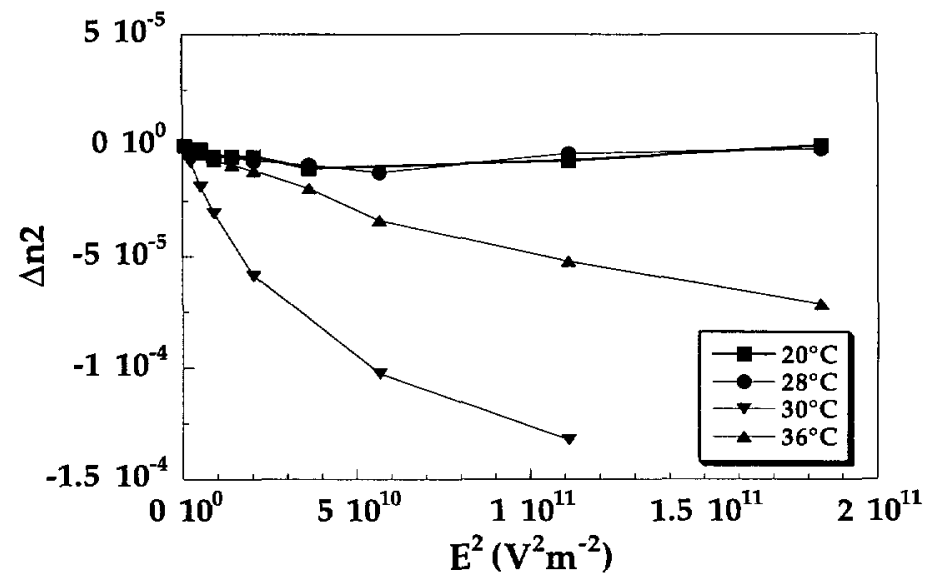

Fig. 6. - Variation of $\Delta n_{2}$ as a function of the square of the applied field, $E^{2}$. Different temperatures are considered while the surfactant concentration is fixed at $1.5 \%$.

\section{- $\Delta n_{3}$ : The Very Slow Positive Response to the Applied Field}

This very slow component of the birefringence, $\Delta n_{3}$, also has a positive sign as $\Delta n_{1}$, but with a much longer ( $\geq 500 \mu \mathrm{s}$ ) characteristic time. As shown in Figure 3d, in the semi-dilute regime, $\Delta n_{3}$ increases sharply in the weak fields and then saturates at stronger fields. The dependence of $\Delta n_{3}$ for a fixed small field $(E=850 \mathrm{~V} / \mathrm{m})$ as a function of concentration is plotted in Figures 4 and $5 c$. This signal appears only for the entangled regime, $c \geq c^{*}$ and it is, therefore, related with the existence of the entanglements. One can interpret such a behaviour using the Edwards tube model [11], which describes usual semi-dilute solutions. The micelles, and hence the tubes, can be strongly oriented under the electric field, due to topological constraints between micelles. In fact, the long measured characteristic time $(\geq 500 \mu \mathrm{s})$ for this component corresponds to tube renewal process coupled to collective rotation: $\Delta n_{3}$ can be associated with the dynamics of a temporary network formed by the entangled micelles.

Unfortunately, it was not possible to perform experiments for even higher concentrations in the semi-dilute regime, because of the high viscosity and the presence of electroconvection in the samples. Thus, the dependence of this long characteristic time on concentration could not be obtained in the semi-dilute regime.

3.2. Temperature Dependence of the Birefringence. - In Figure 7, a typical set of spectra for the same surfactant concentration $(c=1.8 \%)$ is plotted for different temperatures: $26.5^{\circ} \mathrm{C}, 30^{\circ} \mathrm{C}$ and $34^{\circ} \mathrm{C}$. For $c=1.8 \%$ at $26.5^{\circ} \mathrm{C}$, the system is in the semi-dilute regime and the birefringence can be split into two components, while for the same concentration at $30^{\circ} \mathrm{C}$ three contributions to the birefringence are observed. Comparison with Figures $1 \mathrm{c}$ and $1 \mathrm{~d}$ indicates that heating has a similar effect on the spectra as that of decreasing concentration. This fact is coherent with rheological measurements [4] which show that $c^{*}$ is displaced towards higher surfactant concentrations as the temperature increases. Therefore, qualitative changes in the birefringence signals with temperature seem to be related to the displacement of $c^{*}$. 

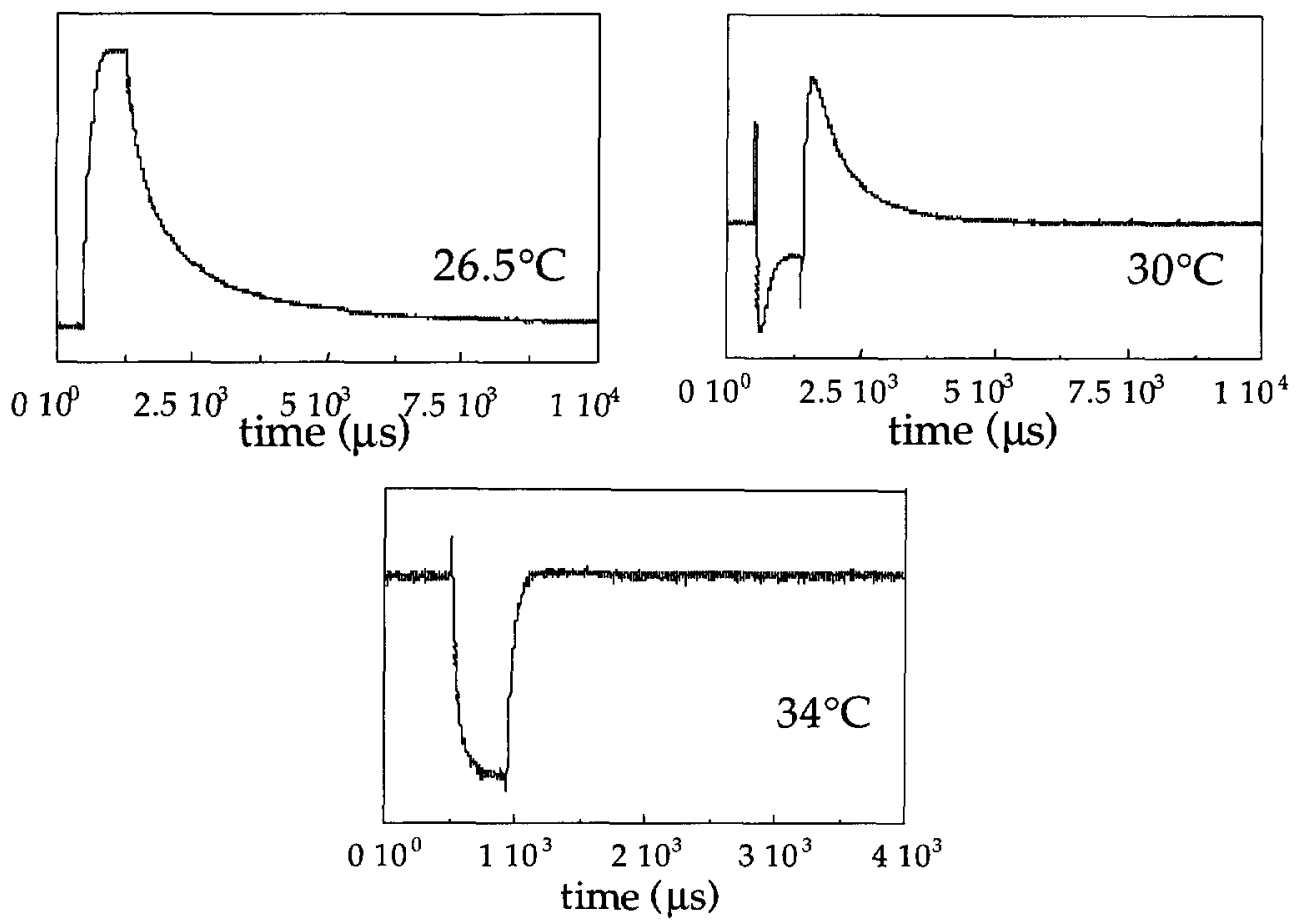

Fig. 7. - Effect of temperature on the spectra of samples containing $1.8 \%$ of surfactant. At $26.5{ }^{\circ} \mathrm{C}$, the solution is in semi-dilute regime.

Following [4], one can quantitatively estimate the dependence of $c^{*}$ on temperature. The theoretical description for the growth of worm-like micelles gives us an estimate for the overlap concentration $[6,7]$ which is:

$$
c^{*} \approx\left(\frac{k_{\mathrm{B}} T l_{\mathrm{B}} a \nu^{2}}{E_{\mathrm{c}}}\right)^{2}
$$

where $E_{c}$ is the end-cap energy, or the energy required to create two end caps from a cylinder. $a$ is the radius of the cylindrical micelle and $\nu$ an effective charge per unit length, $\nu \approx l_{\mathrm{B}}^{-1} l_{\mathrm{B}}=$ $\mathrm{e}^{2} / \varepsilon k T$ is the Bjerrum length, where $\varepsilon$, the dielectric constant of the solution depends of the temperature, $\varepsilon \approx T^{-3 / 2}[5]$. Thus one gets $c^{*} \propto T / E_{\mathrm{c}}^{2}$. For the present case, we consider $c^{*}$ $(T)$ as the surfactant concentration at which the third slow signal corresponding to entangled micelles appears. We obtain:

\begin{tabular}{|l|l|l|l|l|}
\hline$c^{*}$ (weight fraction) & 0.008 & 0.010 & 0.015 & 0.018 \\
$T(\mathrm{~K})$ & 293 & 299 & 303 & 307 \\
$c^{*} / T$ & $2.7 \times 10^{-5}$ & $3.34 \times 10^{-5}$ & $4.95 \times 10^{-5}$ & $5.9 \times 10^{-5}$ \\
\hline
\end{tabular}

It can be seen from the table that the obtained values for $c^{*} / T$ increase with temperature thus the end-cap energy $E_{\mathrm{c}}$ decreases with temperature. 


\section{Conclusions}

Using transient electric birefringence, we investigated the orientational dynamics of charged worm-like micelles in a concentration domain ranging from dilute to semi-dilute condition. We showed that for all studied concentrations the birefringence signal can be split into three different contributions, $\Delta n_{\imath}(i=1,2,3)$, each one with a very different characteristic time. This allowed us to separate the contribution of each component as a function of surfactant concentration. Two of those contributions, $\Delta n_{1}(\tau \approx 5 \mu \mathrm{s})$ and $\Delta n_{3}(\tau \geq 500 \mu \mathrm{s})$ have the same sign, and are related to different orientational mechanisms. The first one, $\Delta n_{1}$, which is the only non-zero contribution in dilute conditions, is related to the orientation of free small micelles in the dilute case. In the semi-dilute regime, this component is also observed and it is related to the rotation of micelles which are smaller than the mesh size, as well as to partial rotation of entangled micelles. In a semi-dilute solution, a much slower component, $\Delta n_{3}$, with the same sign as $\Delta n_{1}$, is also present. This originates from a the release of entanglements between the micelles. A second component, $\Delta n_{2}$, with an opposite sign was observed only in the vicinity of the overlapping concentration $c^{*}$.

One possible interpretation of the anomalous component, $\Delta n_{2}$, has been recently proposed [10] for polyelectrolyte solutions. It states that in those systems a pre-smectic ordering could be present near $c^{*}$. The results presented above on worm-like micellar assemblies are consistent with this disk-like ordering model. The fact that the anomalous effect appears only in a small concentration range near $c^{*}$, the shift of such a concentration domain with temperature, as well as the disappearance of this anomalous signal with the application of very strong fields, seem to corroborate the picture of pre-smectic ordering. The concrete investigations about the relaxation time as well as more detailed signal dependence on the surfactant concentration around $c^{*}$ will be needed.

The complex temperature dependence corresponds actually to the shift of the overlap concentration, $c^{*},[5]$ and a precise analysis of this shift shows that the end-cap energy is a decreasing function of the temperature.

The relaxation of the birefringence signal is in fact, rather intricated, as it presents the relaxation of the three different dynamics overlapped to each other. Also, deriving the $\Delta n$ 's from the plateau values as it is done in this paper, the value we get is rather qualitative. In order to give a quantitative descriptions of this dynamics, one would need to perform the fitting of the relaxation curves. Nevertheless, we believe that the approach of separating the signals in three components would lead us to the correct understanding of dynamics of micelles under electric field around $c^{*}$. Also, further quantitative investigations of the variation of the anomalous component $\Delta n_{2}$ around $c^{*}$ and its dependence on the applied electric field will be necessary. These subjects are presently under investigation and will soon be reported.

\section{Acknowledgments}

The authors are indebted with $\mathrm{P}$. Lemaréchal and C. Fersing who provided strong technical support. Also, we are grateful to $R$. Zana who provided the samples. Discussions with S.J. Candau and Y. Dormoy were enlightening. 


\section{References}

[1] Herb C.A. and Prud'homme R.K., Structure and Flow in Surfactant Solutions, ACS Symposium Series 578, Chap II and Chap III.

[2] Cates M.E. and Candau S.J., J. Phys. Condens. Matter 2 (1990) 6869.

[3] Magid L.Y., Gee J.C. and Talmon Y., Langmuir 6 (1990) 1609-1613.

[4] Zana R. and Talmon Y., Nature 362 (1993) 228.

[5] Kern F., Lequeux F., Zana R. and Candau S.J., Langmuir 10 (1994) 1714.

[6] Safran S., Pincus P., Cates M.E. and Mackintosh F., J. Phys. France 51 (1990) 503.

[7] Mackintosh F., Safran S. and Pincus P., Europhys. Lett. 12 (1990) 697.

[8] Krämer U. and Hoffmann H., Macromolecules 24 (1991) 256.

[9] Hoffmann H., Krämer U. and Thurn H., J. Phys. Chem. 92 (1990) 2027.

[10] Cates M.E., J. Phys. France 2 (1992) 1109.

[11] Doi. M. and Edwards S.F., The Theory of Polymer Dynamics (Clarendon, Oxford, 1986). 